\title{
ERRATA - CORRIGE
}

\author{
(Vedi - Luglio-Agosto-Settembre)
}

M. Pienucci. - Ricerche sperimentali sull'arco elettrico.

\section{ERRATA}

Pag. 90 penultimo rigo

(almeno per intensità non molto grandi)

\section{CORRIGE}

(almeno per intensità non molto grandi) N. B.

N. B. - Per detta proporzionalità osserva che mentre nel 1880 l' Andrews dava la proporzionalità fra l' area e l' intensità $i$ di corrente, il Granqvist nel 1906 da una espressione lineare, rispetto ad $i$, per il raggio del cratere; e che ultimamente (Phil. Mag. 1922, v. 44, pagina 772) il Sellerio trova dei dati per eui appare che i raggii della base negativa e del cratere crescono presso a poco proporzionalmente ad i. A tutto rigore per deboli correnti crescono più rapidamente, per poi crescere meno rapidamente di $i$. 